\title{
incidence de l'anisotropie de consolidation sur le potentiel de liquéfaction statique application au glissement sous-marin du port de Dunkerque
}

\author{
influence of consolidation anisotropy on static \\ liquefaction potentiel \\ application to submarine slope failure in Dunkirk harbour
}

\author{
F. BLONDEAU \\ TERRASOL" \\ Bureau d'Ingénieurs - Conseils en Géotechnique
}

\section{Résumé}

On présente un bref rappel des notions les plus récentes développées dans le domaine de la liquéfaction des sables lâches et notamment la notion de surface critique de déformation par écoulement dans l'espace p,q,e. Lorsque, sous sollicitation non drainée. le chemin de contraintes de l'échantillon atteint cette surface, située à l'intérieur de la surface d'état limite, il se produit une très grande déformation associée à une forte génération de pression interstitielle, dont la conséquence sur un ouvrage peut être analogue à celle d'une liquéfaction.

Plus l'état de contrainte initial de consolidation est anisotrope, plus le risque est grand d'atteindre cette surface sous une sollicitation monotone ou cyclique donnée. En cas de sollicitation monotone, le phénomène résultant est appelé liquéfaction statique ou spontanée.

On présente un cas d'application d'une telle situation au quai à pondéreux ouest (QPO) du port autonome de Dunkerque où deux glissements de talus sousmarins, par liquéfaction spontanée, se sont produits l'un en cours de dragage en 1982. l'autre un an après mise en service en 1984.

\footnotetext{
Abstract

A brief review of the most recent developments about liquefaction of loose sands is given, and particularly the existence of a critical flow deformation surface in the space $p, q, e$ is emphasized. When the stress path of the sample reaches this surface, included inside the limit state surface, under an undrained sollicitation, large deformation occur, associated with high pore pressure development, leading to a phenomenon similar to the liquefaction.

The more anisotropic is the initial consolidation stress state, the higher is the risk to reach such a surface under a monotoneous or cyclic stress. When it is monotoneous, the resulting phenomenon is called static or spontaneous liquefaction.

We present a specific case of application at the western ore quay dock at Dunkirk where two submarine slope failures occured due to spontaneous liquefaction, the first one during the dredging operations in 1982, the second one, one year after achievement in 1984.
}

- Tour Horizon - 52, quai de Dion-Bouton - 92806 Puteaux Cedex. 


\section{INTRODUCTION}

Après un bref rappel sur les notions récentes développées en matière de liquéfaction des sables lâches. On présente un cas d'application de liquéfaction spontanée sous chargement monotone intéressant une pente sous-marine de sables lâches survenue dans le port ouest de Dunkerque.

\section{BREF RAPPEL SUR LA LIQUÉFACTION DES SABLES LÂCHES}

Depuis une vingtaine d'années, les recherches menées sur le potentiel de liquéfaction des sables ont porté, pour la plupart, sur l'aspect cyclique des conditions de chargement et visaient à mettre en évidence l'influence de paramètres tels que :

- densité relative du sable;

- anisotropie de consolidation initiale;

- niveau de contrainte cyclique de cisaillement

- nombre de cycles de chargement.

Plus récemment est apparue la notion de liquéfaction spontanée sous chargement statique ou " liquéfaction statique » pouvant survenir dans des sables lâches soumis à un cisaillement monotone (CASTRO, 1969; CASAGRANDE, 1975; CASTRO, 1975; VAID et FINN, 1979).

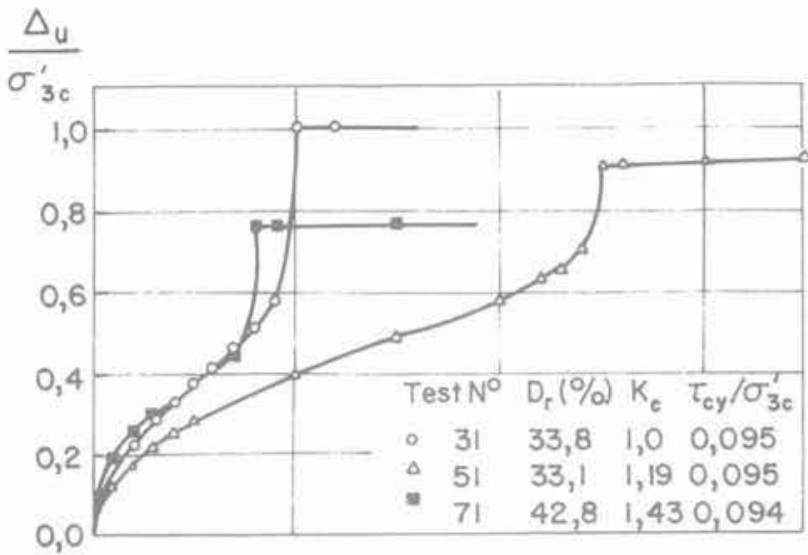

$\epsilon_{1}(\%)$

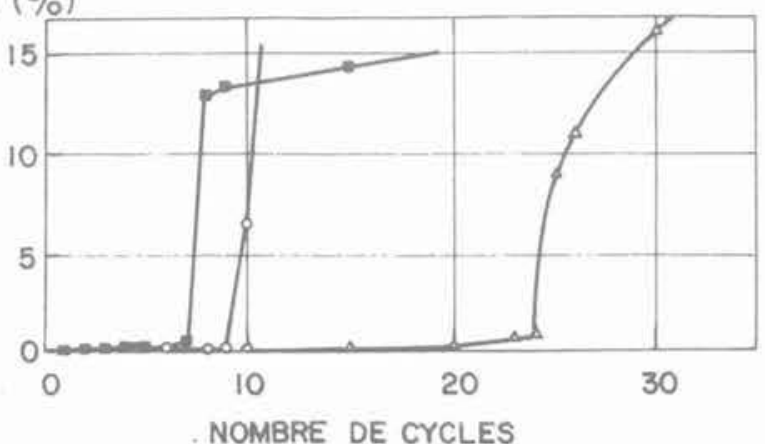

Fig. 1. - Comportement de sables lâches sous chargement cyclique, avec ou sans anisotropie de consolidation initiale (d'après VAID et CHERN, 1983).
Il s'est avéré que les phénomènes constatés au cours d'un tel chargement sont tout à fait semblables à ceux que l'on observe sous chargement cyclique au cours du cycle critique provoquant la liquéfaction (VAID et CHERN, 1982, 1983; SLADEN et al., 1985) et que l'anisotropie de consolidation initiale de l'échantillon est un paramètre déterminant du potentiel de liquéfaction, pouvant jouer dans un sens favorable ou défavorable selon sa valeur.

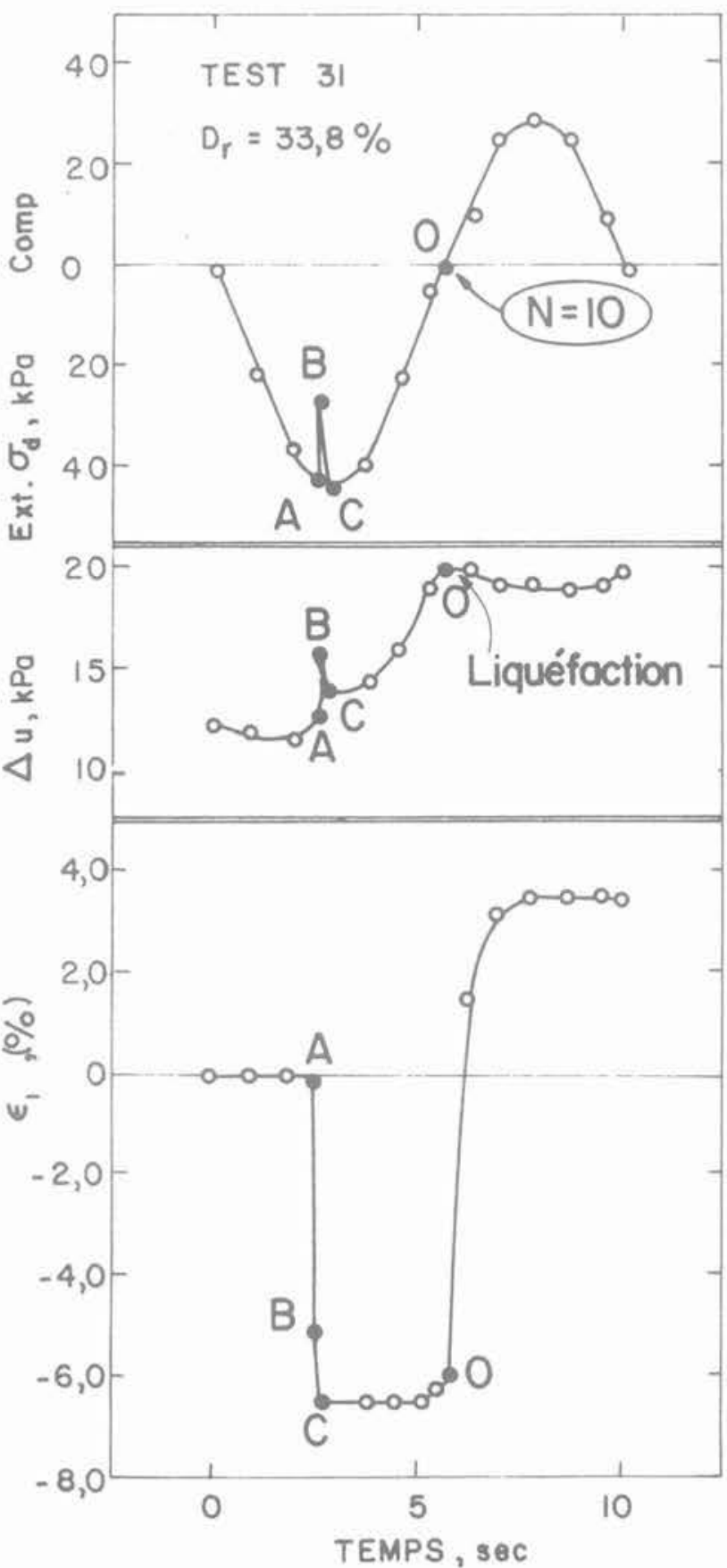

Fig. 2. - Déformation axiale et surpression interstitielle au cours du cycle de dépassement du rapport de contraintes critique (d'après VAID et CHERN, 1982). 

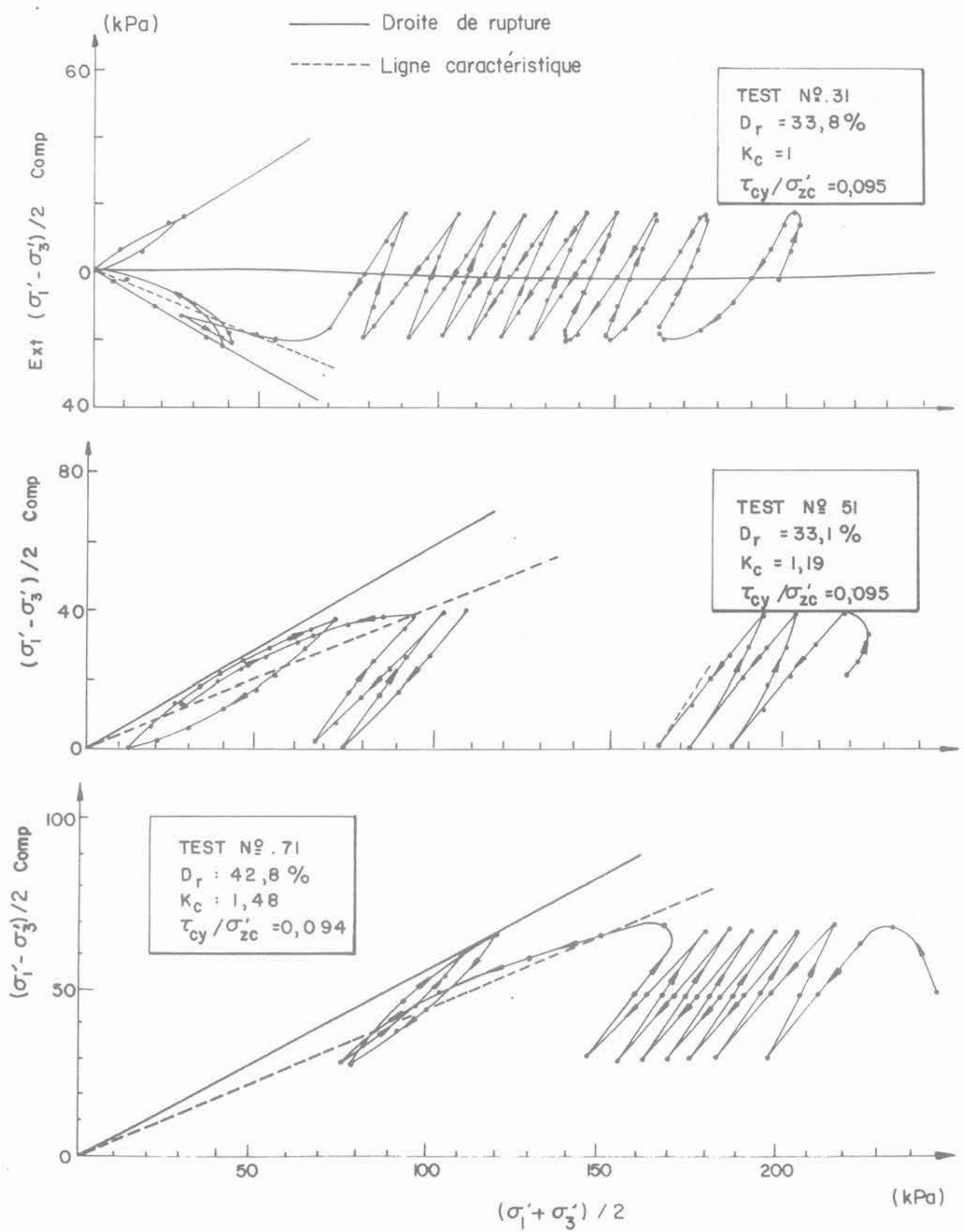

Fig. 3. - Chemins de contraintes au cours d'essais cycliques sur sables lâches (d'après VAID et CHERN, 1982 ). 
La figure 1 montre en effet l'évolution des surpressions interstitielles et de la déformation axiale de trois échantillons de sable lâche de densité relative variant de 33,1 à $42,8 \%$ et soumis à une anisotropie de consolidation initiale. $\mathrm{Ki}=\sigma_{1 \mathrm{c}}^{\prime} / \sigma_{3 \mathrm{c}}^{\prime}$, variant de 1 (état isotrope) à 1,48 , soit $32 \%$ de l'anisotropie provoquant la rupture statique $\left(\Phi=36,6^{\circ}\right)$.

La figure 2 montre l'évolution de ces paramètres au cours du cycle critique de l'un des échantillons:

La figure 3 montre les chemins de contraintes suivis par ces mêmes échantillons durant l'essai. Il apparaît que :

- Dès que le rapport de contraintes principales, $\mathrm{Ki}=\sigma_{1}^{\prime} / \sigma_{3}^{\prime}$, atteint une certaine valeur critique (point A), indépendante de l'état de consolidation initial $\mathrm{Kc}$ ainsi que du taux de cisaillement cyclique, $\sigma_{\text {dcy }} / 2 \sigma_{3 c}^{\prime}$, les échantillons présentent un comportement fortement contractant $(A B)$ conduisant à une augmentation brutale de la pression interstitielle et de la déformation axiale, caractéristique d'un écoulement qui n'est pas la liquéfaction stricto sensu. Dans le plan, $s=\left(\sigma_{1}^{\prime}+\sigma_{3}^{\prime}\right) / 2, \mathrm{t}=\left(\sigma_{1}^{\prime}-\sigma_{3}^{\prime} / 2\right.$, cette valeur critique de $\mathrm{K}$ correspond à deux droites situées à l'intérieur du domaine limité par les droites d'état critique du matériau, appelées droites d'écoulement par VAID et CHERN (1982) et dont SLADEN et al. (1985) ont étendu la notion à celle de surface d'effondrement dans l'espace $\mathrm{p}, \mathrm{q}, e$ de la théorie de l'état critique.

- Immédiatement après, l'échantillon présente un comportement dilatant (BC) avec faible surcroît de déformation axiale, c'est-à-dire arrêt de l'écoulement. Le chemin de contrainte présente un point de rebroussement vers la ligne de rupture.

- L'inversion du sens de chargement provoque à nouveau un comportement fortement contractant dès que le chemin de contrainte repasse à l'intérieur du domaine caractéristique de l'écoulement, mais sans déformation axiale significative.

- Lorque le cisaillement redevient nul, la pression interstitielle atteint sa valeur maximale et provoque une liquéfaction transitoire de l'échantillon. Si le cycle de chargement ne passe pas par la phrase de cisaillement nul, il n'y a pas liquéfaction.

- Les cycles suivants provoquent un chemin de contraintes stabilisé sur la ligne d'état critique avec accumulation de déformation axiale et liquéfaction transitoire à chaque passage sous cisaillement nul.

- Puisque les cycles de chargement se stabilisent sur la ligne d'état critique, la valeur résiduelle atteinte par la pression interstitielle après retour au déviateur initial. rapportée à $\sigma_{3 c}^{\prime}$, décroît en fonction du rapport d'anisotropie initiale $\mathrm{Kc}$ (fig. 1). Elle s'exprime par :

$$
\Delta \mathrm{u}_{\mathrm{res}}=\sigma_{3 c}^{\prime}\left[1-\frac{\mathrm{Kc}-1}{2} \frac{1-\sin \phi^{\prime}}{\sin \phi^{\prime}}\right]
$$

En résumé, les grandes déformations des échantillons de sables lâches ( $\mathrm{Dr} \leqslant 45 \%$ ) proviennent d'une part de la phase d'écoulement générée dès que le chemin de contraintes se situe au-delà de la surface d'effondre- ment, lieu géométrique des pics de cisaillement, et d'autre part des phrases de liquéfaction transitoire provoquée par le passage sous cisaillement nul lors des cycles de chargement ultérieurs.

Cela permet de comprendre pourquoi, plus l'état d'anisotropie initiale est fort, c'est-à-dire plus le point représentatif de l'état de contrainte est proche de la ligne d'écoulement, plus faible est le déviateur cyclique provoquant un certain cumul de déformation axiale après un nombre de cycles donné ( $N=10$ sur la figure 4$)$.
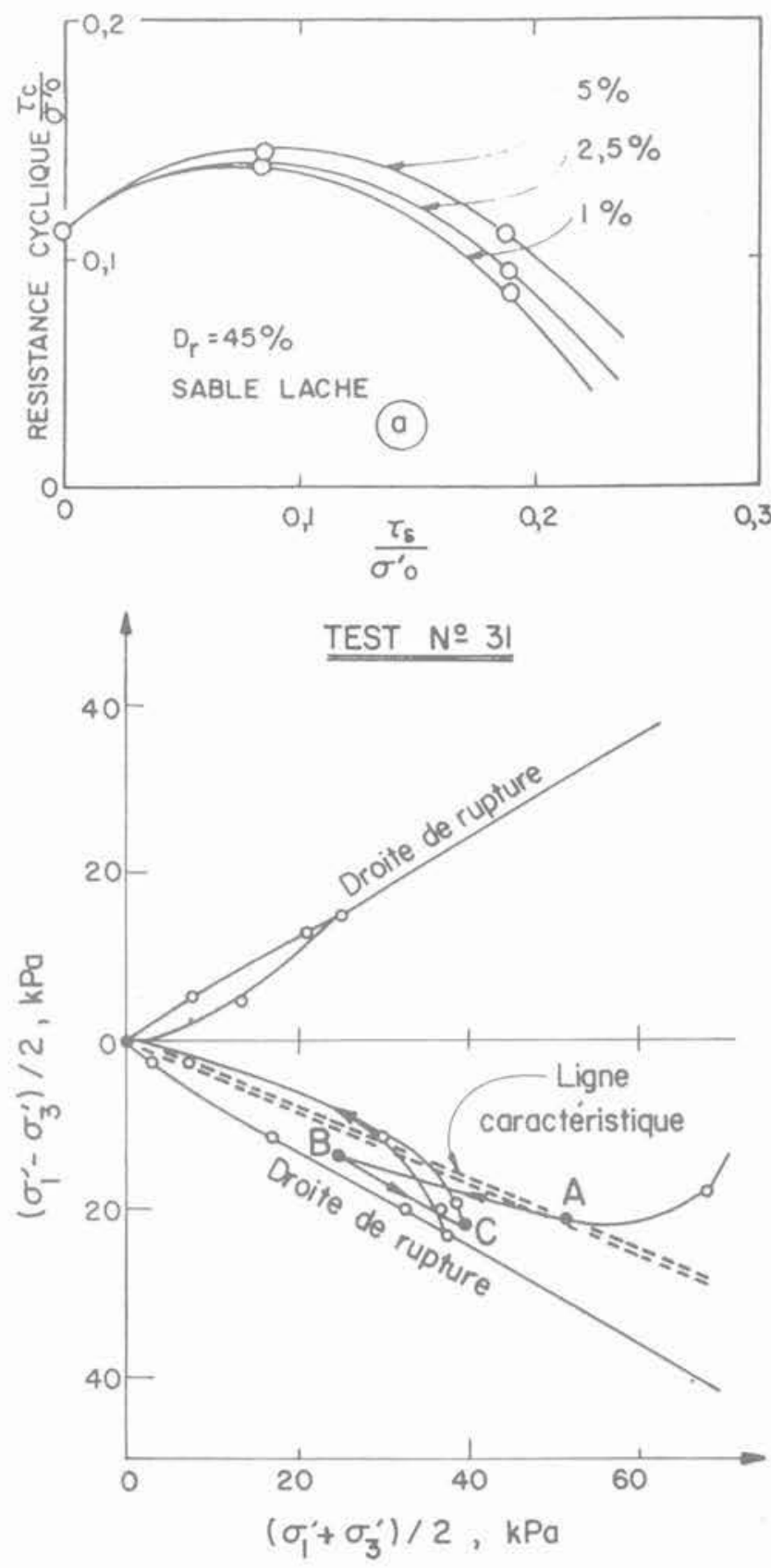

b)

Fig. 4. - Effet d'une contrainte de cisaillement initiale $\tau_{\text {s }}$ sur la résistance au cisaillement cyclique d'un sable lâche definie pour différentes valeurs de la déformation obtenue en 10 cycles (VAID et CHERN, 1983). 
Le phénomène d'écoulement et la notion de surface d'effondrement sont transposables au cas du chargement monotone. La figure 5 présente la synthèse de cette notion. Dans le diagramme s', t, la droite d'écoulement, lieu des pics de cisaillement et trace de l'intersection de la surface d'effondrement avec le plan $e=c^{t e}$, divise le domaine de contraintes admissibles en deux zones distinctes, l'une stable, l'autre instable génératrice de grandes déformations par écoulement.

Le chemin de contraintes 1-2-3 est typique d'un essai non drainé sur sable lâche. L'écoulement apparaît au pic de cisaillement 2. Toute anisotropie de contrainte de consolidation rapprochant l'état initial de la surface d'effondrement diminue la réserve de cisaillement admissible avant écoulement.

On notera que, alors que VAID et CHERN ont considéré que la droite d'écoulement passait par l'origine des axes, SLADEN et al, la font passer par le point critique (steady state point), projection du point d'intersection de la courbe d'état critique et du plan $e=c^{\text {le }}$ correspondant à l'indice des vides de l'échantillon testé.

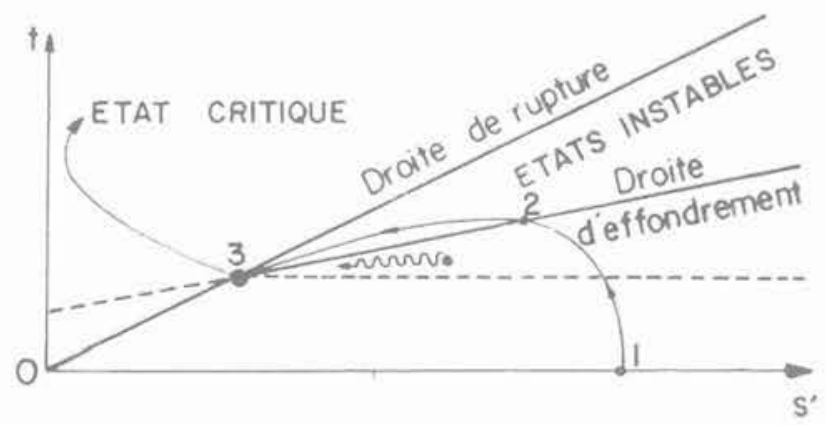

Fig. 5. - Théorie de la surface d'effondrement pour les sables effondrables (d'après SLADEN, 1985).

\section{LA RUPTURE DU BATARDEAU DU QUAI A PONDEREUX OUEST AU PORT DE DUNKERQUE}

Le port de Dunkerque est situé dans l'ensemble des dépôts deltaïques du Rhin. On y rencontre principalement 30 à $40 \mathrm{~m}$ de couches de sables reposant sur le substratum de l'argile des Flandres. La construction des nouveaux bassins se fait par dragage du sable sous l'eau après réalisation des quais à sec.

Deux ruptures importantes de talus sableux sous l'eau se sont produites après la construction du quai à Pondéreux ouest (fig. 6), ruptures dont le mécanisme est lié à la mise en liquéfaction de veines de sable lâche :

$1^{\circ}$ Le 6 novembre 1982 , lors du dragage qui se trouvait à la cote -16 , une rupture s'est amorcée sur le talus Est du bassin. Sa durée a été de 1 h 15 et elle a concerné $300000 \mathrm{~m}^{3}$ de matériau. Le talus avait été taillé à $10 / 1$ dans la partie haute et à $5 / 1$ dans la partie basse. La rupture s'est produite après le dragage d'une couche de limon de $2 \mathrm{~m}$ d'épaisseur située entre les

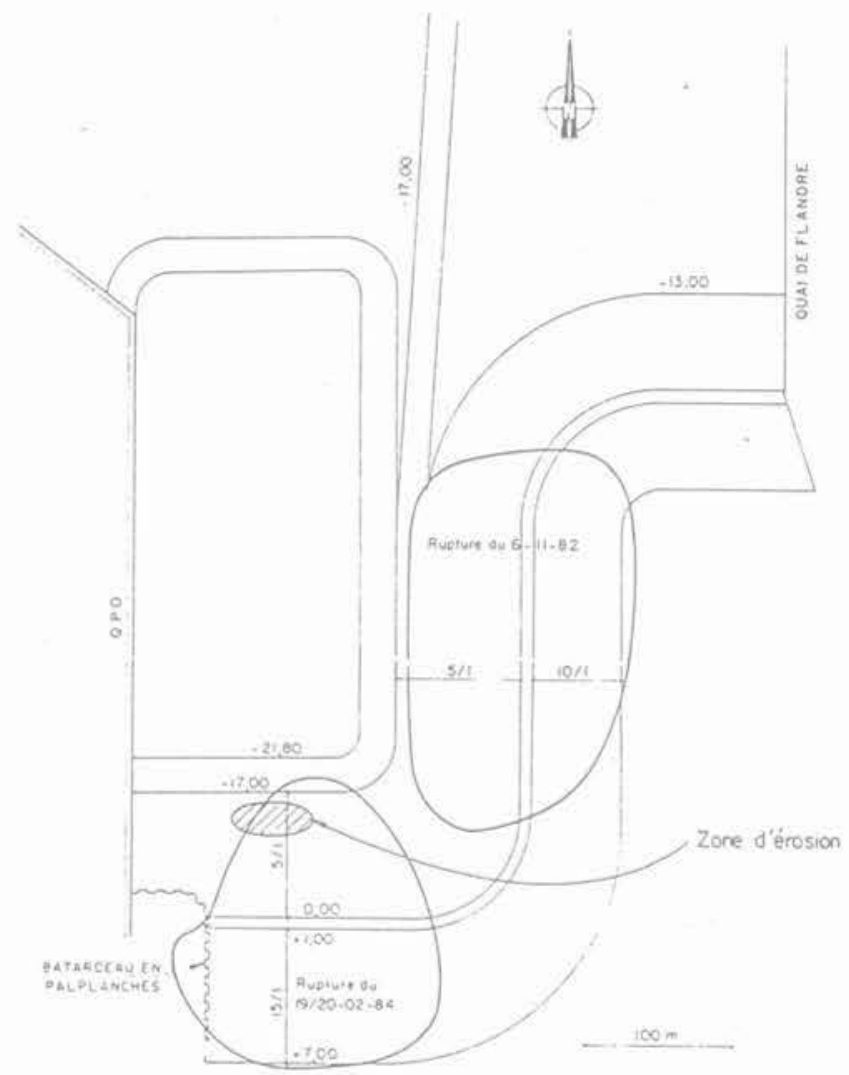

Fig. 6. - Rupture des talus dans le bassin du quai à Pondereux ouest à Dunkerque.

cotes -13 et -15 . A la suite de cette rupture, la pente du talus inférieur a été adoucie à $7 / 1$.

$2^{\circ}$ Dans la nuit du 19 au 20 février 1984, une deuxième rupture s'est produite au fond du bassin dont la construction était terminée depuis un an. Cette rupture a mis en jeu $100000 \mathrm{~m}^{3}$ de matériau et a provoqué la ruine d'un batardeau en palplanches sur $50 \mathrm{~m}$ de longueur.

La figure 7 montre les coupes de la rupture dans le sens principal du mouvement, N-S (7a) et dans le sens perpendiculaire au batardeau (7b). Celui-ci s'est incliné à $45^{\circ}$ environ vers lavant par suite de la suppression de la butée et du dégagement des zones d'ancrage des tirants.

La forme de la surface du 6.11.1982 n'a pas été repérée exactement. Il y a une forte présomption pour qu'elle se soit développée à partir de la base de la couche de limon visible sur les coupes de la figure 7, située à la cote $-15 \mathrm{CM}$, soit 1 mètre au-dessus du niveau de dragage.

La surface de rupture du 19-20.02.1984 a été repérée a posteriori par reconnaissances au pénétromètre statique. Elle affecte la base de la couche de limon sur 50 mètres environ en amont de l'affleurement, puis remonte parallèlement au talus sous-marin, a une dizaine de mètres de profondeur par rapport au fond marin. On remarque sa forme très plate sur 125 mètres de longueur. 
(N)

(s)

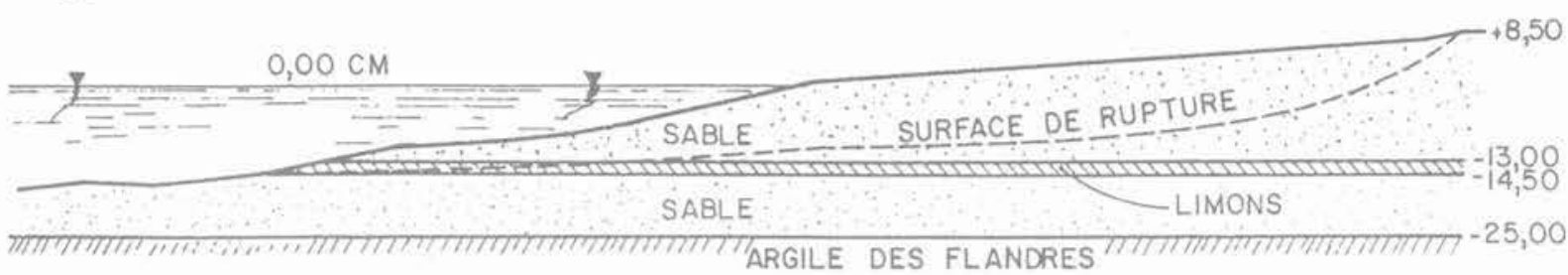

$7 a$

(0)

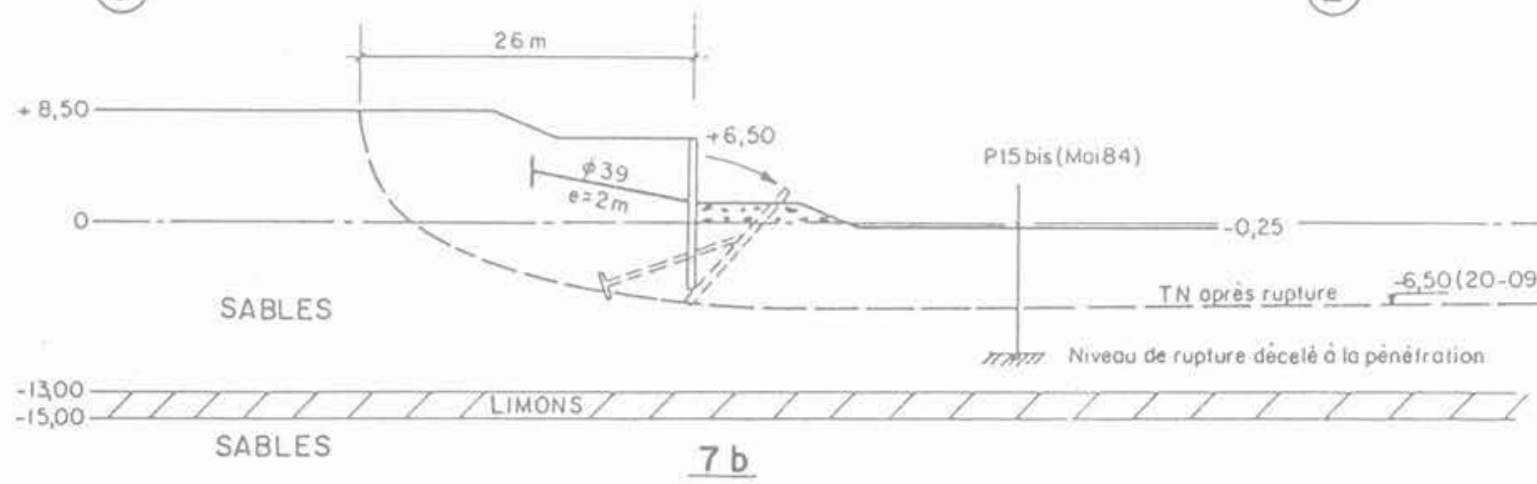

Fig. 7. - Coupes N-S et E-O du site liquéfié du quai Pondereux ouest à Dunkerque.

La stratigraphie (fig. 8), reconnue au pénétromètre statique, est constituée de :

$+8,50$ à $-13 \mathrm{CM}$ Sables coquilliers propres et denses ( $\mathrm{Dr}>60 \%$ en moyenne), peu perméables $\left(\mathrm{K}<5.10^{-6} \mathrm{~m} / \mathrm{s}\right)$.

-13 à $-14,5$ Limons sableux avec intercalation de lits sableux décimétriques $\left(\mathrm{K}<10^{-7} \mathrm{~m} / \mathrm{s}\right)$.

$-14,5$ à $-15 \quad$ A la base de la couche de limons sableux, on trouve une couche de sables lấches de 40 à 50 centimètres d'épaisseur $\left(2<\mathrm{q}_{<}<8 \mathrm{MPa}\right.$ $-20 \%<D_{r}<30 \%$ ) apparemment continue sur le site puisqu'on la retrouve dans deux forages distants de plus de $400 \mathrm{~m}$. Elle serait à l'origine de la liquéfaction.

-15 à $-16 /-18$ Sables et limons graveleux moyennement denses $(40 \%<$ $\mathrm{D}<70 \%)$ et perméables ( $\mathrm{K} \sim$ $3.10^{-4} \mathrm{~m} / \mathrm{s}$ ).

$-16 /-18$ à

$-23 /-25$

La densité relative des sables a été déterminée à partir des corrélations de Schmertmann appliquées au pénétromètre statique.

La perméabilité a été déterminée par essais Lefranc.

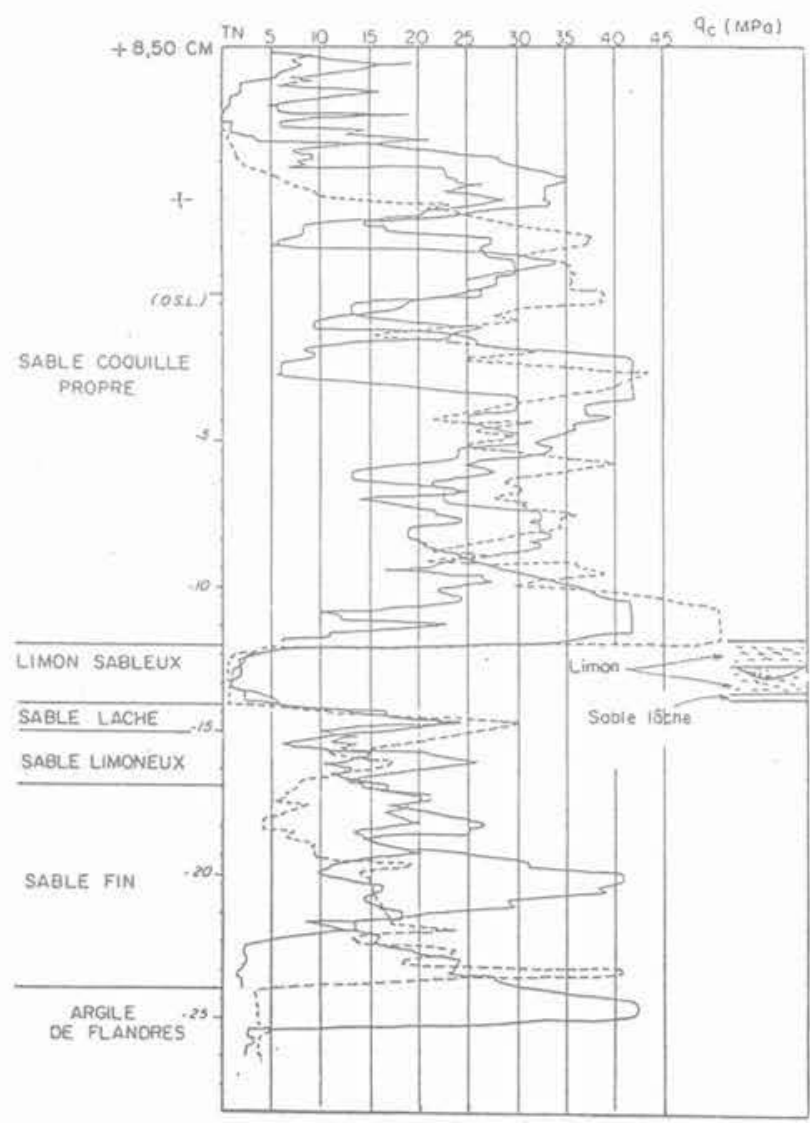

Fig. 8. - Profils pénétrométriques et stratigraphie des couches. 
Une reconnaissance fine au piézocône, avec mesure de surpressions interstitielles de fonçage et mesure du temps de dissipation à certains niveaux d'arrêt de la pointe piézocônique a permis de préciser la susceptibilité des différents sables à la liquéfaction. La figure 9 (a et b) montre les résultats obtenus au voisinage de la couche de limon pour les 4 paramètres mesurés, sur deux sites distants de 400 mètres :

- Pression interstitielle : $\mathrm{u}$

- effort de pointe : $\mathrm{q}_{\mathrm{c}}$

- indice de frottement latéral FR $\left(=q_{s} / q_{\text {}}\right)$

- temps de dissipation des surpressions interstitielles caractérisé par $T_{80}$, délai au bout duquel $80 \%$ des surpressions sont dissipées.

Le tableau I présente les valeurs caractéristiques de ces paramètres que résume la figure 10 . Les trois points relatifs à la base de la couche de limon, constituée de sables lâches, ressortent bien par la forte valeur des surpressions interstitielles développées $\left(\Delta u / u_{0}>3\right)$ et le faible temps de dissipation $(80 \%$ des surpressions dissipées en moins de 10 minutes).

On note, en complément de ces résultats, que les sables de la couche supérieure (au-dessus des limons), bien que qualifiés de denses, ont parfois présenté des surpressions de fonçage non négligeables. Cela est à rapprocher de leur faible perméabilité et de leur comportement fragile après rupture, décelé au pressiomètre autoforeur. La résistance de pointe au pénétromètre pourrait être due plus à une cimentation fragile qu'à une densité relative réellement forte. Ils seraient alors sensibles à un phénomène de rupture en grande masse dès lors que celle-ci est générée en partie aval de talus.

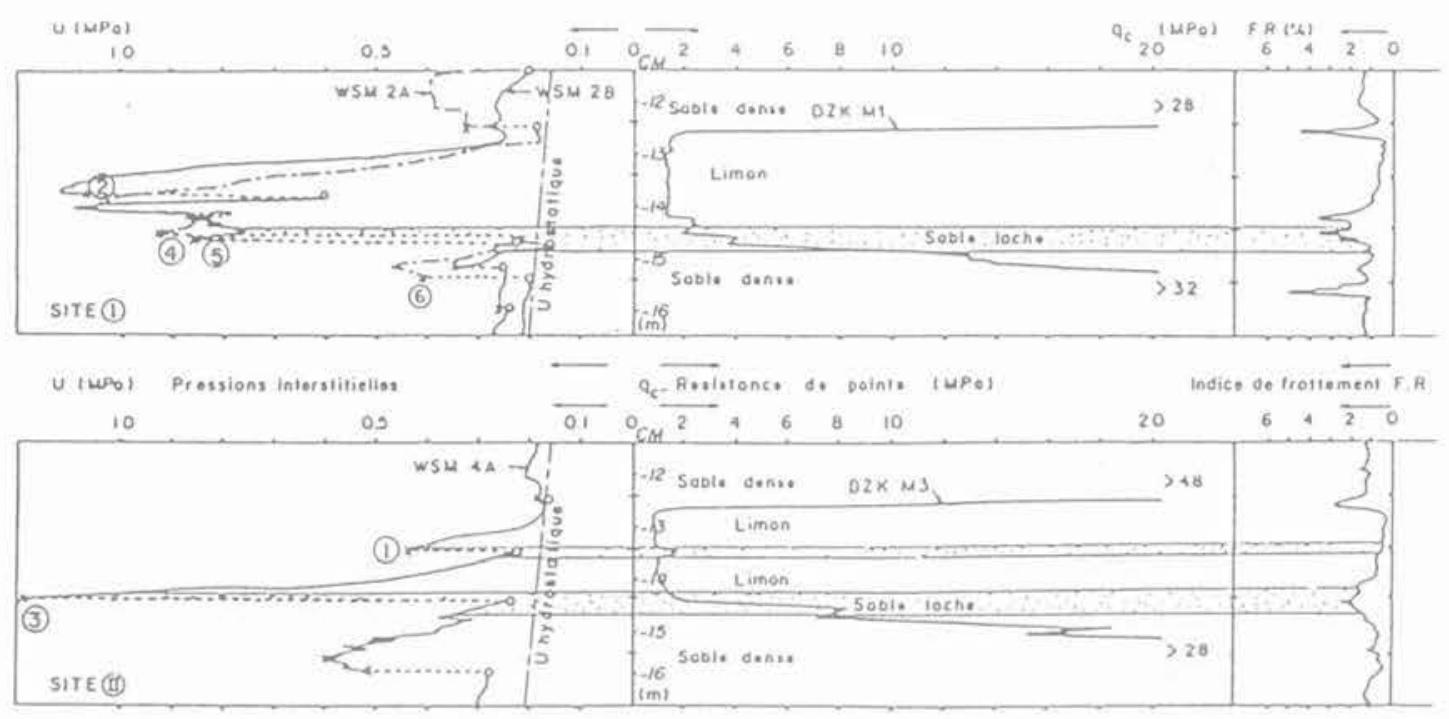

$9 a$

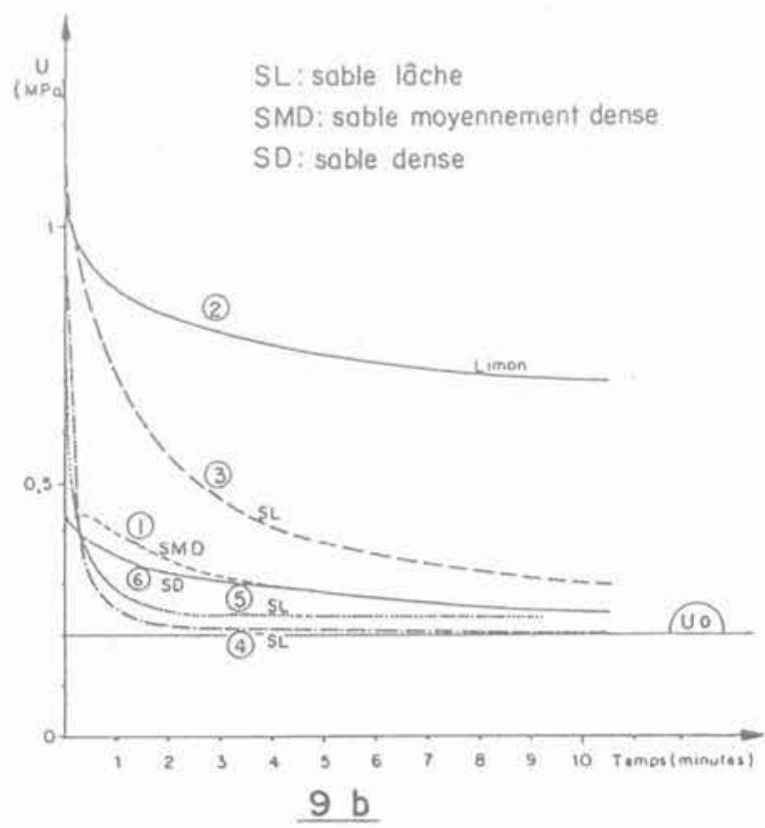

Fig. 9. - Stratigraphie de la couche de limons sableux déduite du piézocône. 
A l'inverse, les sables sous-jacents à la couche de limon, relativement perméables, n'ont pas développé de supression de fonçage, bien que qualifiés de lâches. Ils sont, de ce fait, moins liquéfiables que ne le laisserait penser la seule considération de la densité relative.

Tableau 1.

\begin{tabular}{|c|c|c|c|c|c|}
\hline & Sol & $\Delta \mathrm{u}(\mathrm{MPa})$ & $\Delta \mathrm{u} / \mathrm{u}_{0}$ & $\mathrm{~T}_{80}(\mathrm{~min})$ & $\mathrm{q}_{c}(\mathrm{MPa})$ \\
\hline 1 & Sable limoneux & 0,25 & 1,25 & 10 & $<2$ \\
\hline 2 & Limon & 0,80 & 4,00 & $>600$ & $<2$ \\
\hline 3 & Sable lâche & 0,90 & 4,50 & 5 & 2 à 8 \\
\hline 4 & Sable lâche & 0,70 & 3,50 & 0,5 & 2 a 8 \\
\hline 5 & Sable lâche & 0,65 & 3.25 & 0,5 & 2 à 8 \\
\hline 6 & Sable lấche & 0,21 & 0,10 & 10 & $>30$ \\
\hline
\end{tabular}

Principaux paramètres mesurés au piézocône au voisinage de la zone de rupture.

Les caractéristiques mécaniques classiques de ces matériaux sont excellentes puisque l'on a déterminé :

$$
\begin{array}{llll}
\text { limons: } & c^{\prime}=0 & \phi^{\prime} \text { moyen }=39^{\circ} & \mathrm{Cu} \simeq 45 \mathrm{kPa} \\
\text { sables : } & c^{\prime}=0 & \Phi^{\prime} \text { moyen }=40^{\circ} &
\end{array}
$$

De ce fait, les calculs de stabilité conduisent à des coefficients de sécurité excluant toute explication du sinistre par une rupture statique classique ( $\mathrm{F}>1,6$ pour le batardeau; $F>5$ pour les talus sous-marins).

Par ailleurs, aucun mouvement particulier de l'eau dans le bassin n'a été noté avant la rupture qui s'est produite lors d'une grande marée forte mais pas exceptionnelle (coefficient 110).

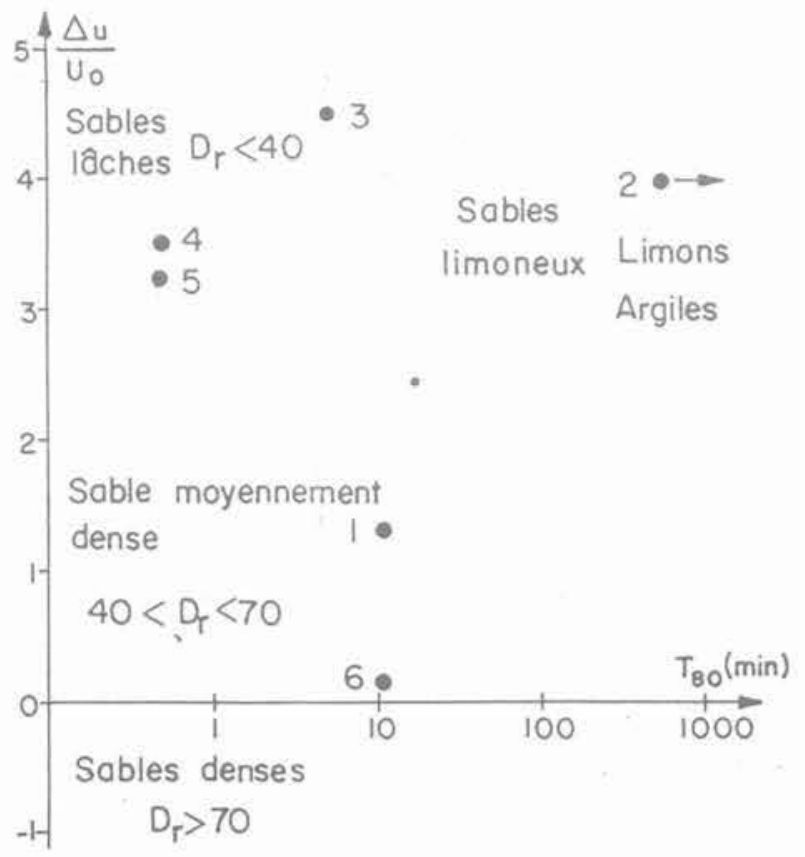

Fig. 10. - Paramètres de génération et dissipation des pressions interstitielles au voisinage de la zone de rupture.
La seule explication plausible est celle d'une liquéfaction statique, spontanée, provoquée par un choc localisé au voisinage de la base de la couche de limons.

Des essais triaxiaux consolidés non drainés pratiqués sur des sables de granulomérie et de densité relative comparables (Port de Nice) avaient conduit au diagramme de H.B. SEED présenté figure 11, dans lequel :
$\tau_{0}=$ contrainte de cisaillement initiale s'exerçant en fin de consolidation sur le plan de rupture, potentiel, de l'échantillon (incliné à $\pi / 4+\Phi / 2$ sur lhorizontale).
$\sigma_{o}^{\prime}=$ contrainte normale initiale s'exerçant sur ce même plan.
$\tau_{i}=$ contrainte de cisaillement s'exerçant sur ce plan au moment du début de l'écoulement (pic du déviateur)

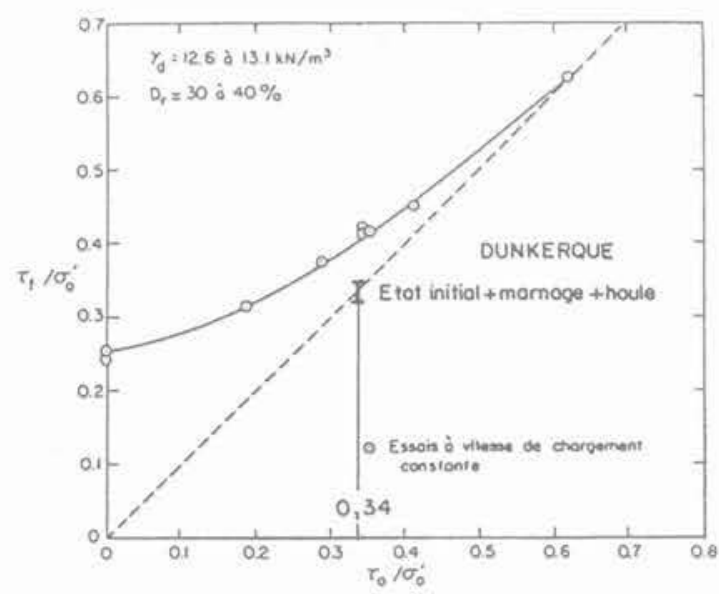

Fig. 11 - Incidence de l'anisotropie de consolidation initiale sur la réserve de cisaillement avant écoulement.

Dans ce diagramme, le point représentatif à l'état de contrainte initiale in situ au voisinage du talus est indiqué ainsi que l'effet du marnage dû à la marée et à une houle de $1 \mathrm{~m}$ d'amplitude en surface, soit $0,50 \mathrm{~m}$ d'amplitude à la base des limons. On a ainsi :

$$
\tau_{\circ} / \sigma_{0}^{\prime}=0,34 \pm 0,016
$$

Dans le cas présent, compte tenu de la valeur de $\tau_{0} / \sigma_{0}^{\prime}$, l'incidence des mouvements d'eau dans le bassin est négligeable vis-à-vis de la liquéfaction. Elle ne laurait pas été pour un talus deux fois plus raide par exemple.

Par contre, une variation de $20 \%$ de ce taux de cisaillement, l'amenant à une valeur proche de :

$$
\tau_{f} / \sigma_{0}^{\prime}=0,40
$$

est tout à fait envisageable sous l'effet d'un effondrement local du talus sous-marin à l'affleurement du limon. Elle suffit à provoquer la liquéfaction spontanée.

Un tel effondrement a pu se produire dans une zone d'érosion significative, observée au cours des mois qui 
ont précédé la rupture, érosion qui avait fait reculer l'affleurement du limon d'une vingtaine de mètres sur 50 mètres de longueưr. Cette zone d'érosion est située au pied de la zone liquéfiée (figure 6).

\section{CONCLUSION}

De nombreux cas de rupture de talus sous-marins ont pu être expliqués par un phénomène de liquéfaction spontanée provoquée par une variation de taux de cisaillement brutale, plus ou moins forte, s'exerçant sur une formation de sables láches liquéfiables. Plus l'anisotropie de consolidation initiale est forte, plus la réserve de cisaillement avant liquéfaction est faible.

Une variation brusque du plan d'eau de quelques mètres peut, par exemple, suffir à provoquer la liquéfaction spontanée d'un talus suffisamment raide de sable lâche. Dans le cas particulier du Port de Dunkerque, où les talus étaient doux $(1 / 10)$, cette cause a été écartée. L'origine du glissement sous-marin qui a emporté $100000 \mathrm{~m}^{3}$ dans la nuit du 19 au 20 février 1984 est plus vraisemblablement un glissement très localisé dans une couche de limon de 2 mètres d'épaisseur reposant sur une couche décimétrique de sables lâches dont l'affleurement avait été largement entamé par érosion sous-marine lors des mois précédant la rupture.

\section{BIBLIOGRAPHIE}

CASAGRANDE A. (1975), Liquefaction and cyclic deformation of sands. A critical review. Proc. 5th. Panamerican Conference on Soil Mech, and Found. Eng. Vol. 5, 79-133.

CASTRO G. (1969), Liquefaction of sands. Ph. D. Thesis. Harward Soil Mechanics Series, $n^{\circ} 81$.

CASTRO G. (1975), Liquefaction and cyclic mobility of saturated sands. ASCE 101 GT6, 551-569.

KROEZEN M., VELLINGA P., LINDENBERG J., BURGER A.M. (1982), Geotechnical and hydraulic aspects with regard to seabed and slope stability. 2nd Canadian Conference on Marine Geotechnical Engineering. Halifax, 1982.

SCHLOSSER F. (1985), Liquéfaction de veines de sables lâches dans des talus sous-marins. 11th Int. Conf. on Soil Mech. and Found. Eng. San Francisco. SEED H.B. (1983), Stability of port fills and coastal deposits, 7th Asian Regional Conference on Soil Mech. and Found. Eng. Vol. 2, 31-41.

SLADEN J.A., D'HOLLANDER R.D., KRAHN J. (1985). The liquefaction of sands, a collapse surface approach. Can. Geot. JI. Vol. 22, n 4, 564-578. VAID Y.P., CHERN J.C. (1982), Mechanism of deformation during cyclic undrained loading of saturated sands. Conference on soil dynamics and earthquake engineering. Southampton, 101-115.

VAID Y.P., CHERN J.C. (1983), Effect of static shear on resistance to liquefaction. Soils and foundations. Vol. $23, n^{\circ} 1,47-60$

VAID Y.P., FINN W.D.L. (1979), Static shear and liquefaction potential. ASCE 105 GT10, 1233-1246. 